\title{
Die jodometrische Bestimmung von Arsen und Antimon neben Kupfer.
}

Von

F. H. Heath. ${ }^{1}$

Es ist angegeben worden, dafs das jodometrische Verfahren zur Bestimmung von Kupfer ohne Fehler in Gegenwart von Arsen und Antimon angewendet werden kann, wenn sich die letzteren Elemente in ihrer höheren Oxydationsstufe befinden. Diese Feststellung rührt von A. H. Low ${ }^{2}$ her, welcher auch sorgfältige Anweisungen zur vollständigen Oxydation des Arsens und Antimons gab. Es scheint jedoch keinerlei Bericht darüber vorzuliegen, ob man versucht hat, Arsen und Antimon jodometrisch zu bestimmen, nachdem man das Kupfer aus dem Gemisch der drei Elemente abgeschieden hat.

Es schien deswegen der Mühe wert zu sein, den Versuch zu machen, Arsen und Antimon im Filtrat vom Cuprojodid zu bestimmen nach der Titration des freien Jods mit Thiosulfat. Zu diesem Zwecke sollten Arsen und Antimon durch Kochen mit Kaliumjodid und schwefliger Säure nach dem Verfahren von Goocr und GRUENER $^{3}$ reduziert und sodann mit einer Normallösung von Jod wieder oxydiert werden. Die Reaktionen, die bei diesen Verfahren vorkommen, können durch die allgemeinen Formeln:

$$
\begin{aligned}
& \mathrm{M}_{2} \mathrm{O}_{5}+4 \mathrm{HJ}=\mathrm{M}_{2} \mathrm{O}_{3}+\mathrm{H}_{2} \mathrm{O}+2 \mathrm{~J}_{2} \\
& \mathrm{M}_{2} \mathrm{O}_{3}+4 J+2 \mathrm{~K}_{2} \mathrm{O}=\mathrm{M}_{2} \mathrm{O}_{5}+4 \mathrm{KJ}
\end{aligned}
$$

zum Ausdruck gebracht werden.

Die Versuche zur Ausführung des Verfahrens wurden mit Brechweinstein und Kaliumarsenat angestellt. Man löste den Brech-

${ }^{1}$ Aus dem Amer. Journ. Sci. (Sill.) ins Deutsche übertragen von J. Koppet-Berlin.

2 Journ. Amer. Chem. Soc. 24, 1083.

${ }^{3}$ Amer. Journ. Sci. 42, Sept. 1891. 
weinstein in Wasser und oxydierte das Antimon durch Normaljodlösung in Gegenwart von Natrium- oder Kaliumbicarbonat. Die erforderliche Jodmenge war ein Mals des vorhandenen Antimons. Die so erhaltene Antimon- oder die Arsenlösung in der höheren Oxydationsstufe wurde angesäuert und mit einer Normallösung von Kupfernitrat versetzt. Das Kupfer bestimmte man jodometrisch nach den früher von Gooch und HEaTH ${ }^{1}$ empfohlenen Vorschriften.

Besondere Vorsicht war nötig bei der Auswahl der Säure, welche man bei der Bestimmung des Kupfers zusetzte. Mineralsäuren dürfen nicht vorhanden sein, weil sie Anlals geben können zu einer Reduktion von Arsen und Antimon durch das überschüssige Kaliumjodid, das man beim Ausfällen des Kupfers hinzufügt. Eine derartige Reaktion würde zu hohe Resultate beim Kupfer und zu niedrige Resultate beim Arsen und Antimon bedingen. Ein Gemisch von Essigsäure und Kaliumjodid reduziert die höheren Oxydationsstufen nur langsam. Weinsäure ist in ihrer Wirkung etwas unregelmäfsig und man läuft dabei Gefahr, dafs sie einen störenden Niederschlag von saurem Kaliumtatrat hervorruft. Günstiger ist die Wirkung von Citronensäure. Die aus einem Gemisch von Citronensäure und Kaliumjodid durch eine Verbindung des fünfwertigen Arsens frei gemachte Jodmenge ist merklich, wenn die Lösung stehen bleibt, während beim Antimonsalz die Reaktion nicht annähernd so schnell verläuft.

Es wurden einige wenige Bestimmungen ausgeführt, um die Wirkung der Citronensäure quantitativ zu prüfen. Bestimmte Mengen von Brechweinstein oxydierte man durch $J_{\text {od }}$ in Bicarbonatlösung, säuerte hierauf die Lösung mit Citronensäure an, fügte gewogene Menge Citronensäure und Kaliumjodid hinzu und liefs bestimmte Zeiten stehen, um festzustellen, ob eine Reduktion stattfand oder nicht. Wenn man die Lösung nach Oxydation mit Jod ansäuerte, wurde etwas Jod frei gemacht, welches man mit einem Tropfen Thiosulfat vor Fortsetzung des Versuches entfernte. Für die Prüfung mit Arsen kam eine Kaliumarsenatlösung von bekanntem Gehalt zur Anwendung. Die Ergebnisse dieser Versuche sind in Tabelle 1 zusammengestellt.

Aus diesen Resultaten ergibt sich, dals bei der jodometrischen Bestimmung von Kupfer neben Arsen das Jod unverzüglich titriert werden mufs, selbst wenn man als Säure die Citronensäure an-

1 Amer. Journ. Sci. 24 (1907) 65; Z. anorg. Chem. 55, 119. 
Tabelle 1.

Reduktionswirkung eines Kaliumjodid-Citronensäuregemisches auf Arsen- und Antimonsalze.

\begin{tabular}{c|c|c|c|c|c|c}
\hline \hline $\begin{array}{c}\mathrm{Sb} \\
\text { angew. } \\
\mathrm{g}\end{array}$ & $\begin{array}{c}\mathrm{As}, \text { an- } \\
\text { gew. als } \\
\mathrm{KH}_{2} \mathrm{AsO}_{4} \\
\mathrm{~g}\end{array}$ & $\begin{array}{c}\text { Angew. } \\
\text { Citronen- } \\
\text { säure } \\
\mathrm{g}\end{array}$ & $\begin{array}{c}\text { Angew. } \\
\mathrm{KJ} \\
\mathrm{g}\end{array}$ & $\begin{array}{c}\text { Vol. der } \\
\text { Lösung } \\
\mathrm{ccm}\end{array}$ & $\begin{array}{c}\text { Zeitdauer } \\
\text { des } \\
\text { Stehens } \\
\text { Min. }\end{array}$ & $\begin{array}{c}\text { Gef. } \\
\text { Jod }\end{array}$ \\
\hline \hline 0.1209 & - & 3 & 4 & 60 & 10 & $\begin{array}{c}\text { k. Farbe } \\
\text { m. Stärke }\end{array}$ \\
0.0797 & - & 3 & 2 & 50 & 20 & $"$ \\
0.1057 & - & 3 & 3 & 60 & 20 & $"$ \\
- & - & - & - & - & - & - \\
- & 0.1238 & 2 & 3 & 50 & 10 & 0.0009 \\
- & 0.1238 & 2 & 4 & 60 & 10 & 0.0012 \\
- & 0.1238 & 3 & 3 & 50 & 10 & 0.0019 \\
- & 0.1238 & 3 & 5 & 75 & 10 & 0.0017 \\
- & - & - & - & - & - & - \\
0.1211 & 0.1238 & 3 & 5 & 100 & 10 & 0.0010 \\
0.1211 & 0.1238 & 3 & 5 & 100 & 10 & 0.0017
\end{tabular}

gewendet hat. Anderenfalls kann Reduktion von Arsensäure unter Auftreten von Jod stattfinden, wodurch das Resultat der Kupferbestimmung beeinflufst wird. Die Antimonsäure wird in mäfsigen Zeiträumen unter ähnlichen Bedingungen nicht reduziert.

Im Laufe der Vorarbeiten zeigte sich, dafs die Tetrathionsäure, die bei der Titration des freien Jods durch Natriumthiosulfat bei der Kupferbestimmung gebildet wird, die folgenden Operationen stört. Es bildet sich nämlich beim Kochen der Lösung nach Zusatz von Schwefelsäure freier Schwefel und Schwefelwasserstoff, indem die Tetrathionsäure Zersetzung erleidet. Hierdurch können Sulfide von Antimon und Arsen ausgefällt werden. Es wurden verschiedene Mittel versucbt, die Tetrathionsäure und ihre Zersetzungsprodukte vor Reduktion von Arsen und Antimon zu oxydieren. $\mathrm{Zu}$ diesem Zweck kamen festes Jod und Kaliumnitrit zur Anwendung, jedoch ohne vollen Erfolg. Bei Benutzung von Bromwasser jedoch oder noch besser von flüssigem Brom waren die Ergebnisse zufriedenstellend.

Es zeigte sich, dals nach Zusatz hinreichender Mengen von flüssigem Brom zur kalten Lösung nach Zersetzung des ganzen Überschusses des vorhandenen Kaliumjodids und darauffolgendes Kochen der Lösung die Tetrathionsäure nur noch sehr geringe 
Störungen verursacht. Zur Zersetzung des bei der Fällung von Kupfer im Überschufs zugesetzten Kaliumjodids wendete man ungefähr $1 \mathrm{ccm}$ Brom an. Wurde die Lösung dann nach kurzem Kochen beim Abkühlen nicht klar, so setzte man etwas mehr Brom zu, um vollständige Oxydation zu sichern und kochte weiter, um den Bromüberschufs zu vertreiben. Es ist zweckmälsig, in diesem Stadium die Lösung etwas zu konzentrieren, um die Hauptmenge des Broms zu entfernen. Andernfalls kann bei Zusatz von Kaliumjodid für die Reduktion des Arsen- oder Antimonsalzes so viel von diesem Reduktionsmittel zersetzt werden, dals die Reduktion nicht mehr vollständig ist. In der Lösung blieb nach der Reduktion etwas freies Jod und es erwies sich als schwierig, dies in heifser Lösung mit Genauigkeit zu entfärben. In kalter Lösung konnte Stärke als Indikator angewandt werden; demnach wurde in der abgekühlten Lösung das freie Jod in Gegenwart von Stärke durch einen Überschul's von schwefliger Säure entfärbt. Hierauf verdünnte man die Lösung auf $100 \mathrm{ccm}$ oder mehr und setzte Jodlösung bis zur schwachen Färbung zu. Die blalsblaue Farbe entfernte man dann durch sorgfältigen Zusatz verdünnter schwefliger Säure aus einer Pipette. Die Lösung wurde hierauf durch Natrium- oder Kaliumbicarbonat neutralisiert and Arsen oder Antimon durch Titration mit Normaljodlösung in der üblichen Weise bestimmt. Die folgenden Tabellen zeigen die bei diesem Verfahren erhaltenen Ergebnisse.

(S. Tabelle $2-4$, S. 91 u. 92.)

Das Verfahren zur Bestimmung von Kupfer und Arsen oder von Kupfer neben Antimon ist also das folgende:

Eine Lösung, die Kupfer und Arsen oder Antimon in ihrer höchsten Oxydationsform enthält, wird mit $1-2 \mathrm{~g}$ Citronensäure versetzt. Zur Fällung von nicht über $0.3 \mathrm{~g}$ Kupfer in einem $V_{0}$ lumen von $50 \mathrm{ccm}$ werden $3 \mathrm{~g}$, und in einem Volumen von $100 \mathrm{ccm}$ 5 g Kaliumjodid verwendet. Das freie Jod titriert man mit Natriumthiosulfatlösung. Die Gleichungen, die hierbei in Betracht kommen, sind folgende:

$$
\begin{aligned}
& 2 \mathrm{CuSO}_{4}+4 \mathrm{KJ} \rightarrow 2 \mathrm{~K}_{2} \mathrm{SO}_{4}+\mathrm{Cu}_{2}+\mathrm{J}_{2} \text { und } \\
& 2 \mathrm{~J}+2 \mathrm{Na}_{2} \mathrm{~S}_{2} \mathrm{O}_{3}=2 \mathrm{NaJ}+\mathrm{Na}_{2} \mathrm{~S}_{4} \mathrm{O}_{6} .
\end{aligned}
$$

Nach der Titration wird das Cuprojodid auf Asbest abfiltriert und das Filtrat nach Zusatz von $1 \mathrm{ccm}$ flüssigem Brom in einem Erlenmeyerkolben gekocht, wobei man einen Verschlufs anwendet, um Verlust durch Spritzen zu verhindern. 
Tabelle 2.

Kupfer und Arsen.

\begin{tabular}{|c|c|c|c|c|c|c|c|c|c|}
\hline 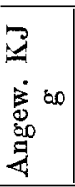 & 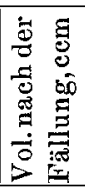 & 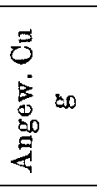 & مه & 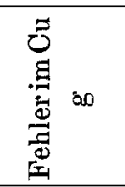 & 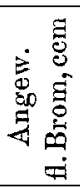 & 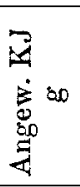 & 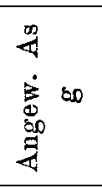 & 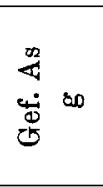 & 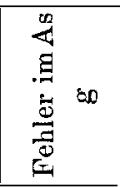 \\
\hline 3 & 50 & 0.0700 & 0.0700 & \pm 0.0000 & 1.0 & 2 & 0.1238 & 0.1231 & -0.0007 \\
\hline 3 & 50 & 0.0700 & 0.0693 & -0.0007 & 1.0 & 2 & 0.1238 & 0.1231 & -0.0007 \\
\hline 3 & 50 & 0.0875 & 0.0869 & -0.0006 & 1.0 & 2 & 0.1238 & 0.1239 & +0.0001 \\
\hline 4 & 50 & 0.0700 & 0.0698 & -0.0002 & $\left\{\begin{array}{l}0.6 \\
0.4\end{array}\right\}$ & 1 & 0.1238 & 0.1235 & -0.0003 \\
\hline 4 & 45 & 0.0700 & 0.0703 & +0.0003 & 1.0 & 2 & 0.1238 & 0.1247 & +0.0009 \\
\hline 4 & 50 & 0.0700 & 0.0700 & \pm 0.0000 & 1.0 & 2 & 0.1238 & 0.1235 & -0.0003 \\
\hline 5 & 70 & 0.1400 & 0.1407 & +0.0007 & $\left\{\begin{array}{l}1.0 \\
0.5\end{array}\right\}$ & 2 & 0.1238 & 0.1233 & +0.0005 \\
\hline 5 & 50 & 0.0910 & 0.0907 & -0.0003 & $\left\{\begin{array}{l}1.0 \\
0.5\end{array}\right\}$ & 2 & 0.1238 & 0.1239 & +0.0001 \\
\hline 4 & 50 & 0.0700 & 0.0703 & +.00003 & 1.0 & 2 & 0.1238 & 0.1237 & -0.0001 \\
\hline 4 & 50 & 0.0875 & 0.0879 & +0.0004 & 1.0 & 2 & 0.1238 & 0.1234 & -0.0004 \\
\hline 5 & 65 & 0.1400 & 0.1410 & +0.0010 & 1.0 & 3 & 0.1238 & 0.1239 & +0.0001 \\
\hline 3 & 30 & 0.0875 & 0.0860 & -0.0015 & 0.8 & 2 & 0.0495 & 0.0493 & -0.0002 \\
\hline
\end{tabular}

Tabelle 3.

Kupfer und Antimon.

\begin{tabular}{|c|c|c|c|c|c|c|c|c|c|}
\hline $\begin{array}{l}3 \\
80 \\
\sum_{0}^{\circ 0} \\
\sum_{4}^{\circ}\end{array}$ & 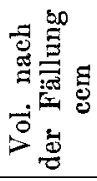 & $\begin{array}{l}\overrightarrow{0} \\
\dot{0} \\
\dot{a} \\
\dot{\pi}\end{array}$ & $\begin{array}{l}\overrightarrow{0} \\
\dot{0} \\
\dot{0} \\
\dot{0}\end{array}$ & 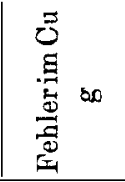 & 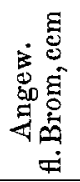 & 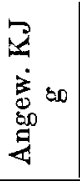 & 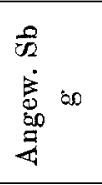 & 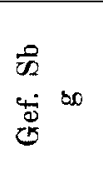 & 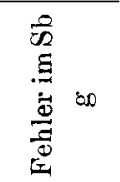 \\
\hline 4 & 85 & 0.0700 & 0.0703 & +0.0003 & $\left\{\begin{array}{l}1.0 \\
0.5\end{array}\right\}$ & 2 & 0.1417 & 0.1421 & +0.0004 \\
\hline 4 & 80 & 0.0700 & 0.0701 & +0.0001 & $\left\{\begin{array}{l}1.0 \\
0.5\end{array}\right\}$ & 2 & 0.1727 & 0.1725 & -0.0002 \\
\hline 4 & 80 & 0.0875 & 0.0869 & -0.0006 & $\left\{\begin{array}{l}1.0 \\
0.5\end{array}\right\}$ & 2 & 0.1286 & 0.1289 & +0.0003 \\
\hline 5 & 80 & 0.0735 & 0.0739 & +0.0004 & $\left\{\begin{array}{l}1.0 \\
0.5\end{array}\right\}$ & 2 & 0.1641 & 0.1645 & +0.0004 \\
\hline 5 & 90 & 0.1050 & 0.1050 & \pm 0.0000 & $\left\{\begin{array}{l}1.0 \\
0.5\end{array}\right\}$ & 2 & 0.1378 & 0.1372 & -0.0006 \\
\hline 4 & 75 & 0.0875 & 0.0874 & -0.0 & $\left\{\begin{array}{l}1.0 \\
0.5\end{array}\right\}$ & 2 & 0.1329 & 0.1326 & -0.0003 \\
\hline 6 & 115 & 0.0700 & 0.0707 & +0.0007 & $\left\{\begin{array}{l}1.3 \\
0.5\end{array}\right\}$ & 2 & 0.2475 & 0.2477 & +0.0003 \\
\hline 8 & 120 & 0.1575 & 0.1571 & -0.0004 & $\left\{\begin{array}{l}1.5 \\
0.5\end{array}\right\}$ & 2 & 0.1419 & 0.1413 & -0.0006 \\
\hline
\end{tabular}

Wenn nach kurzem Kochen und Verdampfen der Hauptmenge des frejen Jods die Lösung nicht klar wird, kühlt man ab, setzt 
etwas mehr Brom $(0.5 \mathrm{ccm})$ hinzu und kocht nochmals. Ist die Lösung klar geworden, so wird sie auf etwa $60 \mathrm{ccm}$ konzentriert, damit alles Brom entweicht. Hierauf wird sie auf $100 \mathrm{ccm}$ verdünnt, mit $2 \mathrm{~g}$ Kaliumjodid versetzt und auf $50 \mathrm{ccm}$ eingekocht. Nach dem Abkühlen der Lösung entfärbt man das Jod durch Zusatz von schwefliger Säure unter Anwendung von Stärke als Indicator, sodann verdünnt man auf $100 \mathrm{ccm}$, setzt wieder Jodlösung bis zur Färbung hinzu und entfärbt genau durch sorgfältigen Zusatz verdünnter schwefliger Säure aus einer Pipette. Nach Neutralisation der Lösung mit Natrium oder Kaliumbicarbonat wird Arsen oder Antimon mit Normaljodlösung in der üblichen Weise titriert.

Nach den angeführten Ergebnissen scheint es möglich, auf diese Weise Kupfer und Arsen oder Kupfer und Antimon mit einem Fehler von nur wenigen Zehnteln Milligramm zu trennen und zu bestimmen. Es ist gleichfalls möglich, die Summe von Arsen und Antimon mit ziemlicher Genauigkeit festzustellen und Kupfer von

\section{Tabelle 4.}

Kupfer, Arsen und Antimon.

\begin{tabular}{|c|c|c|c|c|c|c|c|c|c|c|c|c|c|}
\hline $\begin{array}{l}3 \\
3 \\
0 \\
3 \\
z\end{array}$ & 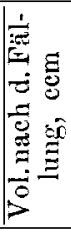 & 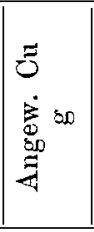 & 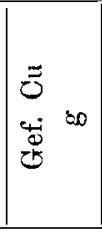 & 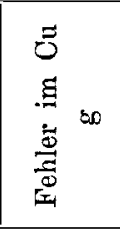 & 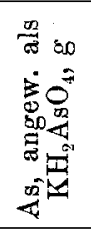 & 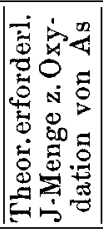 & 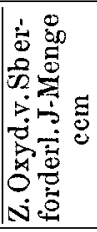 & 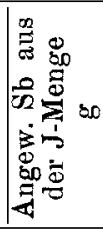 & 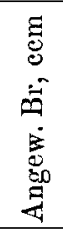 & 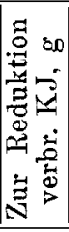 & 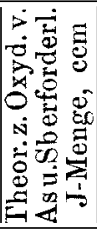 & 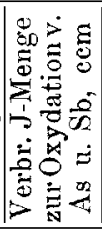 & 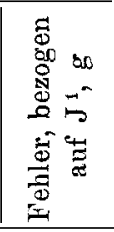 \\
\hline - & - & $\ldots$ & - & - & 1238 & 32.54 & 17.87 & 0.1088 & 1.0 & 2 & 50.41 & 50.06 & -0 \\
\hline 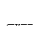 & - & - & - & - & 95 & 13.01 & & 0. & 1.0 & 2 & 47 & 46 & -0 \\
\hline 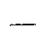 & - & - & - & - & 38 & 32.54 & 0 & 8 & 0.5 & 2 & 94 & 47.70 & -0 \\
\hline - & - & - & - & - & 0.0990 & 27.21 & 2 & 34 & 1.0 & 2 & 33 & 90 & -0 \\
\hline$\tilde{j}$ & 90 & 0.0700 & 0.0718 & 8 & 5 & .01 & 5 & 0.1044 & & 2 & .16 & 0.00 & \\
\hline 5 & 90 & 0.0700 & 0.0710 & & 0.0990 & 27.21 & 86 & & & 2 & 9.07 & 9.00 & 009 \\
\hline $5^{2}$ & 95 & 0.1222 & 0 & & 0 & 27.21 & & & $\left\{\begin{array}{l}1.5 \\
0.3\end{array}\right\}$ & 2 & .89 & 6.23 & 81 \\
\hline 9 & 115 & 875 & & & & 27.21 & & & 2.0 & 3 & & 10 & - \\
\hline 6 & 110 & & & & & 27.21 & & & 1 & 2 & & 42.64 & -0 \\
\hline $7^{2}$ & 100 & 613 & 0.1611 & 0.0002 & 0.0990 & 27.21 & 18.92 & 0.1101 & 1.5 & 2 & 46.13 & 45.90 & -0.0028 \\
\hline
\end{tabular}

${ }^{1}$ Gew. von Jod $\times 0.50=$ Fehler, vollständig berechnet auf Sb.

" " $" \times 0.30=, \quad " \quad$, As.

${ }^{2}$ Es wurden besondere Mengen Kupfer ausgewogen. 
liesen beiden zu trennen und zu bestimmen. Im letzteren Falle zann gleichfalls die Summe von Arsen und Antimon bestimmt werden; aber die so erhaltenen Werte für Kupfer werden etwas zu roch und die für Arsen und Antimon etwas zu niedrig (Tabelle 4).

Zum Schlufs möchte der Verfasser Herrn Prof. F. A. GoocH für die freundliche Unterstützung und die vielen Ratschläge im Laufe dieser Untersuchung seinen Dank aussprechen.

New Haven, U.S. A., The Kent Chemical Laboratory of Yale University.

Bei der Redaktion eingegangen am 6. Mai 1908 . 